\title{
Structures of High and Low Density Amorphous Ice by Neutron Diffraction
}

\author{
J. L. Finney, ${ }^{1, *}$ A. Hallbrucker, ${ }^{2}$ I. Kohl, ${ }^{2}$ A. K. Soper, ${ }^{1,3}$ and D. T. Bowron ${ }^{1,3}$ \\ ${ }^{1}$ Department of Physics and Astronomy, University College London, Gower Street, London WC1E 6BT, United Kingdom \\ ${ }^{2}$ Institut für Allgemeine und Anorganische Chemie, Universität Innsbruck, Innrain 52a, A-6020 Innsbruck, Austria \\ ${ }^{3}$ ISIS Facility, Rutherford Appleton Laboratory, Chilton, Didcot, Oxon OX11 OQX, United Kingdom
}

(Received 31 January 2002; published 17 May 2002)

\begin{abstract}
Neutron diffraction with isotope substitution is used to determine the structures of high (HDA) and low (LDA) density amorphous ice. Both "phases" are fully hydrogen bonded, tetrahedral networks, with local order similarities between LDA and ice I $h$, and HDA and liquid water. Moving from HDA, through liquid water and LDA to ice $\mathrm{I} h$, the second shell radial order increases at the expense of spatial order. This is linked to a fifth first neighbor "interstitial" that restricts the orientations of first shell waters. This "lynch pin" molecule which keeps the HDA structure intact has implications for the nature of the HDA-LDA transition that bear on the current metastable water debate.
\end{abstract}

DOI: $10.1103 /$ PhysRevLett.88.225503

The conjecture that there are two forms of water at low temperature has dominated recent debate on the phase behavior of metastable water [1-7]. It has been argued [8] that high density (HDA) and low density (LDA) amorphous ices are physical manifestations of these two postulated forms, with the structure of LDA relating to that of deeply supercooled liquid water at ambient pressure [9], while that of HDA relates to that of the denser liquid water that is found at higher pressure $[9,10]$.

These views are being increasingly questioned. For example, it has been argued [11-13] that the formation of HDA by pressurizing ice $\mathrm{I} h$ arises from a breakdown of the mechanical stability of the ice lattice which results in a heavily deformed and disordered crystalline ice rather than a genuinely glassy phase. Other spectroscopic work [14] is interpreted as suggesting that neither LDA nor HDA have liquidlike structures, the absence of sharp powder diffraction peaks not necessarily excluding the presence of disordered microcrystalline material [14]. Likewise Johari [15] recently proposed that "HDA may be a mixture of highly strained microcrystalline high-pressure polymorphs of ice." These arguments call into question the postulated thermodynamic connection between these two putative amorphous phases and liquid water, and hence reflect on the controversial hypothesis of the existence of two critical points in water [8].

Despite a significant amount of experimental work $[9,10,16,17]$ structural measurements reported to date for these two materials have been limited to measurements of the total structure factors and total radial distribution functions, $g(r)$, by either $\mathrm{x}$-ray or neutron diffraction $[9,10,16]$. However, a full structural characterization of water requires the three site-site radial distribution functions $g_{\mathrm{HH}}(r)$, $g_{\mathrm{OH}}(r)$, and $g_{\mathrm{OO}}(r)$ to be obtained. Here we present the first experimental determination of these functions for both HDA and LDA, using hydrogen isotope substitution with neutron diffraction.

High density amorphous ice was prepared by pressurizing samples of $\mathrm{H}_{2} \mathrm{O}, \mathrm{D}_{2} \mathrm{O}$, and a $50: 50 \mathrm{H}_{2} \mathrm{O} / \mathrm{D}_{2} \mathrm{O}$ mixture
PACS numbers: 61.43.Fs, 61.12.-q

in a piston cylinder apparatus of $20 \mathrm{~mm}$ inner diameter up to $1.6 \mathrm{GPa}$ at liquid nitrogen temperature. The inner part of the cylinder was lined with indium foil of $0.5 \mathrm{~mm}$ thickness. Each batch of high density amorphous ice was recovered to ambient pressure and small samples taken for characterization by $\mathrm{x}$-ray diffraction and differential scanning calorimetry, to confirm that the appropriate materials had been produced. Each HDA sample was loaded into a parallel sided TiZr cell for data collection at $80 \mathrm{~K}$ on the SANDALS diffractometer of the ISIS pulsed spallation neutron source, UK. The measured neutron diffraction patterns showed no sign of Bragg peaks that would have indicated the presence of crystalline material. After data collection on HDA, the temperature of each sample was raised to $122 \mathrm{~K}$ for the in situ conversion to LDA. The temperature was then reduced and LDA data taken at $80 \mathrm{~K}$. Subsequent spectroscopic analysis of the samples confirmed the isotopic compositions of the $\mathrm{D}_{2} \mathrm{O}$ and 50:50 $\mathrm{H}_{2} \mathrm{O} / \mathrm{D}_{2} \mathrm{O}$ samples, respectively, as $99.9(0.3)$ and 49.6 (0.3) mol \% $\mathrm{D}_{2} \mathrm{O}$. Background, can scattering, multiple scattering, and attenuation corrections were made using the ATLAS data analysis suite [18]. The data were then normalized for powder packing fraction by scaling the HDA and LDA sample scattering against a series of measurements made on $\mathrm{H}_{2} \mathrm{O}, \mathrm{D}_{2} \mathrm{O}$, and $\mathrm{HDO}$ polycrystalline ice $\mathrm{I} h$, formed in situ in the same sample can by freezing water of the appropriate isotopic composition.

In order to allow for exploration of the geometries of the intermolecular structures consistent with the measured partial pair correlation functions, the empirical potential structure refinement (EPSR) procedure [19] was implemented. This process performs a Monte Carlo computer simulation of the system under study to generate ensembles of water molecules whose structures are consistent with the measured diffraction data. This procedure was also applied to previously published partial structure factor data on ambient liquid water at $298 \mathrm{~K}$ and crystalline ice $\mathrm{I} h$ at $220 \mathrm{~K}$ [20]. The water molecule ensembles so obtained are used to calculate structural quantities such as site-site radial 
distribution functions (rdfs, Fig. 1) and spatial density functions (sdfs, Fig. 2). For the respective systems, ice $\mathrm{I} h$, water, LDA, and HDA, the atomic densities used within the structure refinements were $0.0921,0.1002,0.0937$, and 0.1170 atoms $\AA^{-3}$. All of the tests that have been done to date using this procedure [21] indicate strongly that forcing the simulated molecular ensembles to reproduce the measured radial distribution functions is a substantial constraint on the three-body and higher-order correlation functions, and will almost certainly capture the essential topology of the local order. One example, where the robustness of the structures obtained can be seen, is a comparison of the structures determined for a dilute alcohol water solution [22,23]: two independent and differently weighted sets of isotopic substitution data on the same chemical system were both analyzed by the EPSR procedure. The resulting models are found to display the same local order.

The oxygen-oxygen (OO) rdfs for LDA and HDA in Fig. 1 show very similar features to the corresponding x-ray derived rdfs reported in Refs. [16] and [17]. The $\mathrm{x}$-ray curve is effectively the $\mathrm{OO}$ function as $\mathrm{x}$ rays see hydrogen only very weakly. In contrast, neutrons are more heavily weighted towards the hydrogen correlations that are important in understanding the detailed network structures. The consistency between these earlier x-ray results and the work presented here confirms the validity of the neutron scattering corrections and data analysis proce-

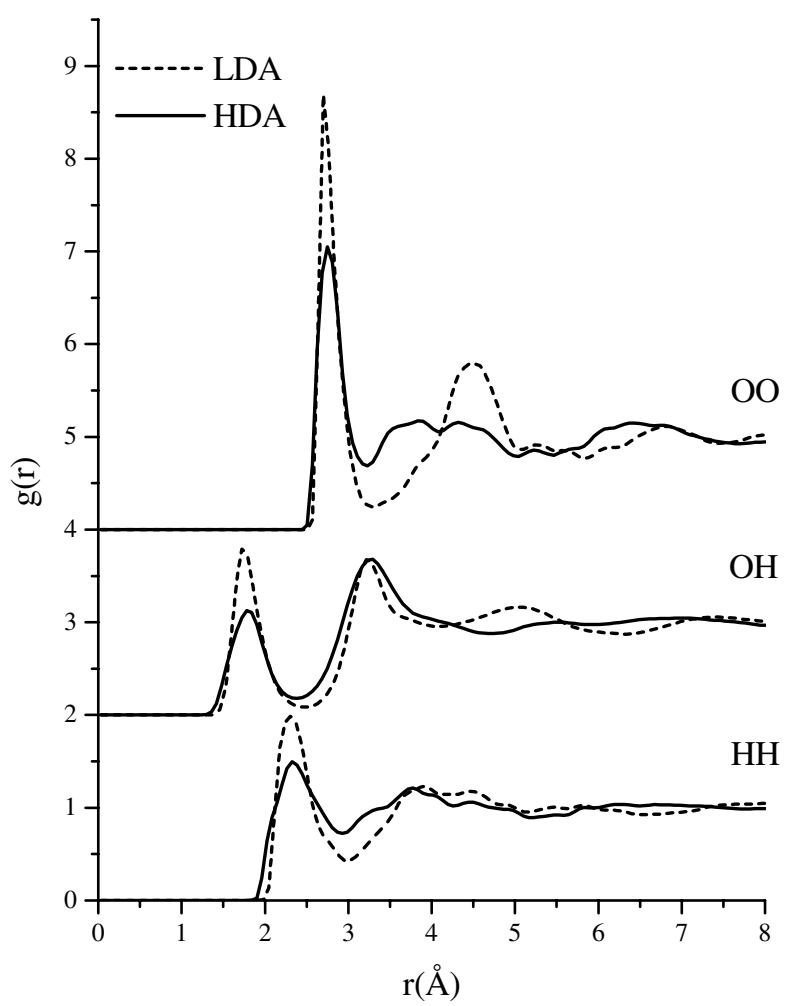

FIG. 1. Intermolecular partial radial distribution functions of HDA and LDA at $80 \mathrm{~K}$. For clarity, the ordinates for $g_{\mathrm{OH}}(r)$ and $g_{\mathrm{OO}}(r)$ are shifted by 2 and 4 , respectively. dures. Peak positions and heights are comparable between the neutron-derived $g_{\mathrm{OO}}(r)$ and the X-ray rdfs for the respective phases. Particularly notable is the broad second peak in the HDA OO partial distribution function, which is clearly resolved into two peaks.

The first neighbor oxygen-hydrogen $(\mathrm{OH})$ coordination number obtained by integrating the $\mathrm{OH}$ partial rdf between 1.4 and $2.5 \AA$ is essentially 2 (0.1) for both LDA and HDA. This is the same for liquid water and ambient pressure crystalline ice, and is evidence for full hydrogen bonding in both amorphous ice structures. Looking beyond the first neighbor peaks, the differences between the HDA and LDA partials indicate that the two structures are clearly different (Fig. 1). The intermolecular coordination numbers give interesting insight into the nature of these differences. First, for the OO data integrated between 2.3 and $3.3 \AA$, we find that LDA has $3.9(0.1)$ nearest neighbors, a number that is consistent within the errors with that found in
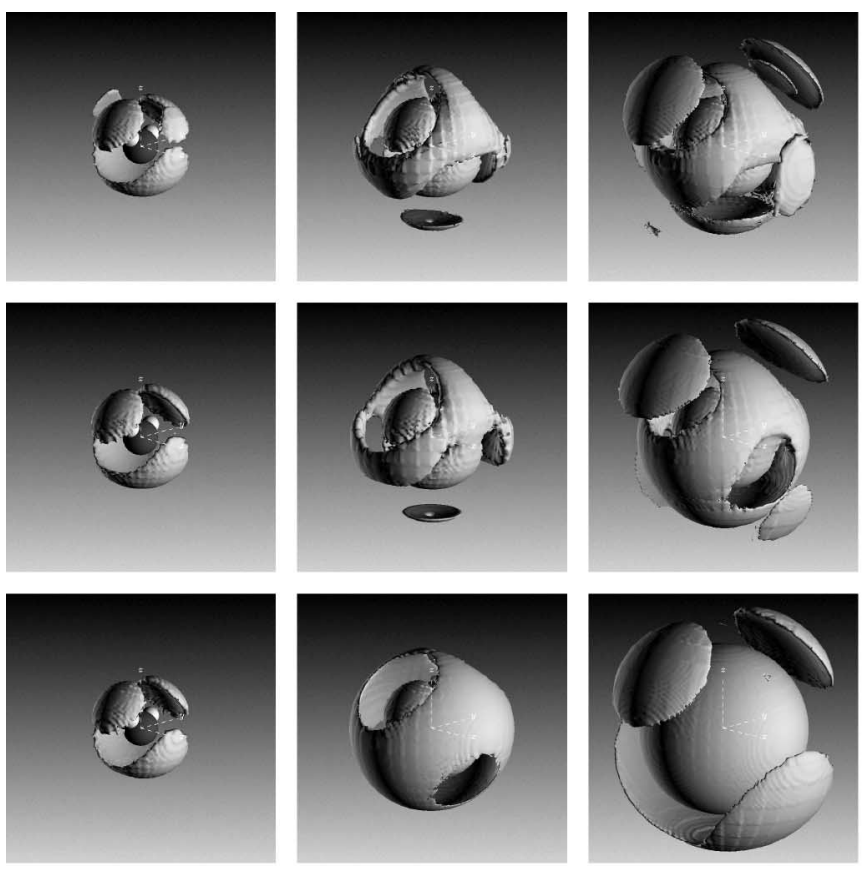

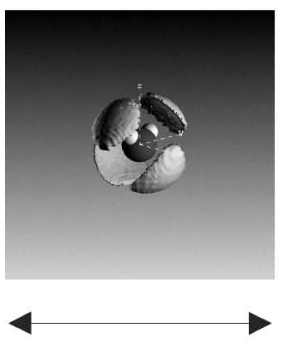

$15 \AA$

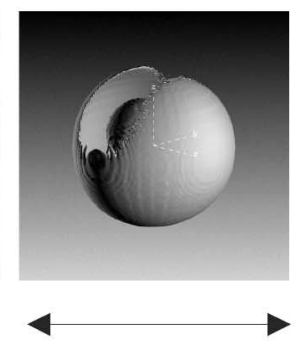

$15 \AA$

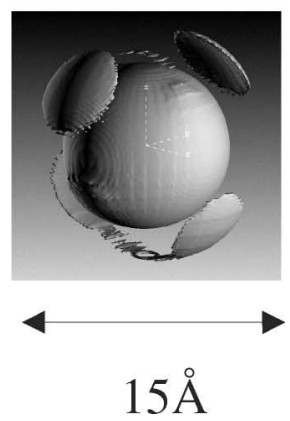

FIG. 2. Spatial density functions showing the distribution of (from left to right) the first, second, and third neighbor water molecules around a central water for (bottom to top) crystalline ice at $220 \mathrm{~K}$ [24], LDA at $80 \mathrm{~K}$, liquid water at $298 \mathrm{~K}$ [24], and $\mathrm{HDA}$ at $80 \mathrm{~K}$. The contour levels $[g(r, \Omega)]$ used are, respectively, $(3.1,2.1,1.5),(2.3,1.7,1.2),(1.7,1.3,1.1),(2.1,1.5,1.2)$. 
crystalline ice at $220 \mathrm{~K}, 3.7$ (0.1). The liquid water value of $4.3(0.12)$ is known from many studies to be slightly but significantly greater than 4.0 , and is thus slightly greater than for either LDA or $220 \mathrm{~K}$ crystalline ice I $h$. In contrast, the interestingly higher value of $5.0(0.1)$ obtained for HDA signifies that an additional water molecule is present in the first neighbor shell and confirms a hypothesis put forward in earlier x-ray work [16]. This raises the interesting question of where in the first coordination shell the additional molecule is, and how it interacts with the molecule at the center of the coordination shell.

Molecular spatial density functions are the clue to locating where this extra molecule is positioned (Fig. 2). Whereas the rdfs are spherically averaged, these spatial functions give directional information about the pair correlations. For the present data on amorphous ice, water, and ice $\mathrm{I} h$, the "normal" first coordination shell picture is seen in all four systems (left hand column of Fig. 2). The two lobes above the central water molecule indicate the location of neighboring molecules that accept hydrogen bonds from the central water. The broad lobe "beneath" the central molecule corresponds to hydrogen bonded neighbors that interact with the central molecule's lone pairs of electrons. The strong similarity of the plots indicates very similar first neighbor structures for all four forms of water, and underlines the hydrogen bonding conclusions drawn above from the $\mathrm{OH}$ coordination numbers. The splitting of the lone pair lobes in ice $\mathrm{I} h$ is indicative of the stronger tetrahedrality forced by the crystallinity of the system.

For HDA we note an additional close-in feature: there are two further lobes that indicate the directions in which the additional "first neighbor" water molecules approach the central molecule. As we have already noted that the first $\mathrm{OH}$ coordination number remains close to 2 for HDA, as in LDA, we can conclude that this additional close water molecule is not hydrogen bonded to the central water molecule. This conclusion is further supported by an examination of the orientational correlation function (not shown) between the central water and those molecules occupying this lobe. The dominant orientation of this molecule is consistent with it being hydrogen bonded only to water molecules other than the central one, as is found for similarly close nonbonded first neighbors in some of the high pressure ices $[24,25]$.

Considering now the second shell spatial distribution functions (middle column, Fig. 2), we note a remarkable similarity between HDA and liquid water. Though spatially similar, it is important to note that the radial distance of this "lobe" is larger in liquid water than in HDA. Thus, although the HDA second shell structure bears considerable resemblance to that of liquid water at $298 \mathrm{~K}$, it occurs at a shorter distance, consistent with the higher atomic density of HDA. We note further from Fig. 2 that structural similarity between HDA and liquid water is evident also in the third shell. In this context, it is interesting to recollect the speculation of Mishima et al. [26] that the formation of HDA could be considered as a "melting" to a liquid at higher pressure. The structural similarities observed here seem to bear out this conjecture quite dramatically. The coordination number of 5.0 observed here for HDA, together with the additional first shell lobe in the spatial distribution function (Fig. 2) are consistent with the suggestion [11-13] of an additional molecule being forced into the first shell.

In contrast, LDA has a markedly different local structure from both HDA and liquid water at $298 \mathrm{~K}$. For example, significantly different second and third neighbor $\mathrm{OO}$ coordination numbers indicate differences in the second and third neighbor environments. Referring to the spatial density functions (Fig. 2), two points stand out. First, the second neighbor shell for LDA (middle panel) is much more uniform than it is either in liquid water or in HDA. Second, a similar second shell diffuseness is also seen in the ice $\mathrm{I} h$ spatial density functions. This indicates that first shell hydrogen-bonded neighbors in LDA and ice $\mathrm{I} h$ are free to adopt a wider range of spatial correlations than those in HDA or ambient water. This infers that the second neighbor molecules have greater orientational freedom within the second shell, correlating with the lower atomic and molecular density of these systems.

Figure 3 shows that this change in the spatial order in the second coordination shell, as we move from HDA, through ambient liquid water and LDA to crystalline ice, is matched by a sharpening of the second neighbor peak in the OO rdf. We note that this change is correlated with the presence in the first neighbor shell of HDA (1.0 molecules on average) and liquid water ( 0.3 molecules on average)

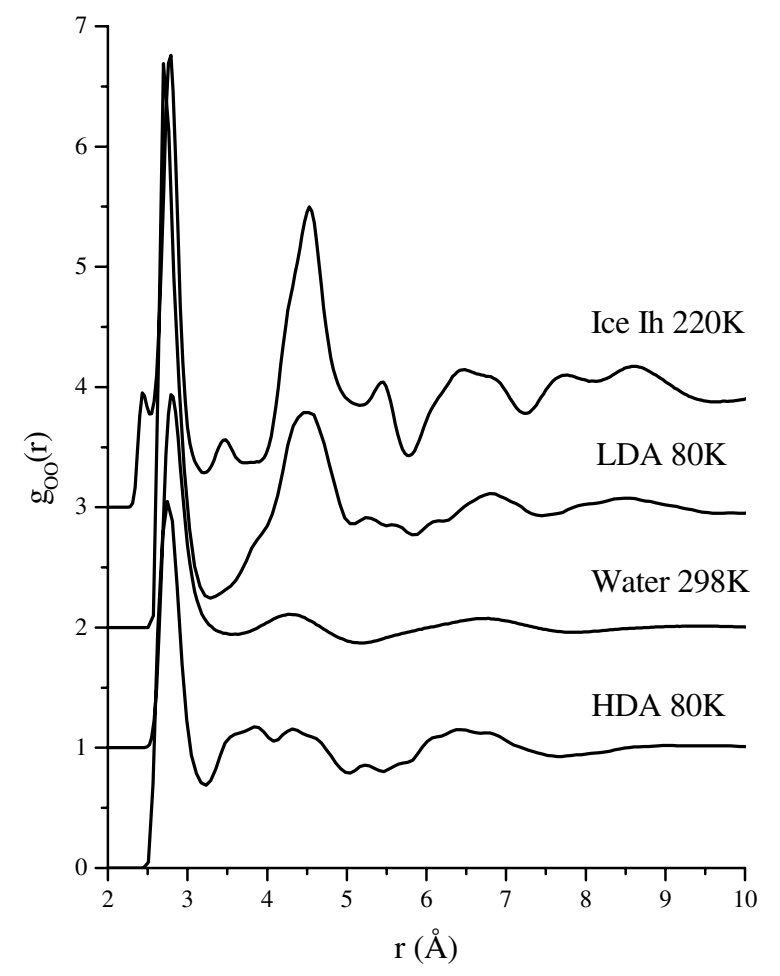

FIG. 3. Oxygen-oxygen partial radial distribution functions of LDA, HDA, liquid water, and ice $\mathrm{I} h$. 
of the additional water molecule that is not directly hydrogen bonded to the central water molecule, as already described. This "interstitial" molecule pins the possible orientations of first shell water molecules, and forces spatial structure onto the second shell. This restriction could occur through either the interstitial's interactions with first shell molecules or those beyond [27]. In LDA and ice I $h$, where there are few if any interstitial molecules, the hydrogen bonded first shell molecules find themselves freer to rotate about their water-water hydrogen bonds. This freedom gives rise to the spatially more diffuse second shell, but interestingly is accompanied by a much higher oxygen atom positional order. Overall these findings demonstrate the degree to which the interstitial water molecule close to the first neighbor shell can influence the local order.

Our results show no evidence for microcrystallinity in our samples of HDA or LDA. Though there is considerable similarity in the local order between LDA and ice I $h$ (Fig. 2), beyond the second shell there are increasing discrepancies between their respective rdfs (Fig. 3) which cannot be explained in terms of a simple lattice distortion or crystallite size effect. To establish this point further we have taken the diffraction data for ice $\mathrm{I} h$ and broadened the peaks assuming a range of crystallite sizes between 15 and $25 \AA$ A. The results demonstrated that to obtain diffraction patterns consistent with those measured for LDA, crystallites smaller than $\sim 15 \AA$ would be needed. This is barely more than the equivalent of two unit cells and does not appear sufficient to support a microcrystalline model of LDA. With the structure factors of HDA being less sharp than for LDA, it follows that the HDA data also cannot be fitted by a realistic microcrystalline model. However, the similarity in local order between LDA and ice I $h$ may well explain the observed similarities [11] between their librational and vibrational densities of state. Out to the second neighbors the structures are comparable, and it is not surprising they have similar excitation spectra for these modes. Thus it does not appear necessary to invoke crystallinity to explain this spectroscopic data.

Referring again to the middle panel of Fig. 2, we see four systems in which the second shell distribution develops progressively from one of relatively high spatial order (HDA) to one of low spatial order (LDA), correlated with the progressive removal of an interstitial water molecule. Closer to ambient conditions the transition from low density water to high density water is continuous as a function of applied pressure [28], and a closely analogous growth of interstitial molecules is observed as the density increases. The transformation HDA to LDA is sudden and can be triggered only by raising the temperature. The present data show clearly the presence of interstitial molecules in HDA, but not in LDA. Therefore the picture implied by these data is that the HDA-LDA transition cannot occur at low temperature because the molecular kinetic energy is insufficient to let the interstitial molecules jump out of their first shell positions. These interstitial molecules therefore act as the lynch pin which keeps the HDA structure intact.

We thank Kevin Knight for discussions, and the ISIS Facility for neutron beam time and support facilities.

*Corresponding author.

Email address: j.finney@ucl.ac.uk

[1] P. H. Poole, F. Sciortino, U. Essmann, and H. E. Stanley, Nature (London) 360, 324 (1992).

[2] E. G. Ponyatovskii, V. V. Sinand, and T. A. Pozdnyakova, JETP Lett. 60, 360 (1994).

[3] C. T. Moynihan, Mater. Res. Soc. Symp. Proc. 455, 411 (1997).

[4] O. Mishima and H. E. Stanley, Nature (London) 392, 164 (1998).

[5] C. A. Jeffrey and P. H. A. Austin, J. Chem. Phys. 114, 484 (1999).

[6] P. H. Poole, F. Sciortino, T. Grande, H. E. Stanley, and C. A. Angell, Phys. Rev. Lett. 73, 1632 (1994).

[7] S. Sastry, P. G. Debenedetti, F. Sciortino, and H. E. Stanley, Phys. Rev. E 53, 6144 (1996).

[8] O. Mishima and H. E. Stanley, Nature (London) 396, 329 (1998).

[9] M.-C. Bellisent-Funel, J. Teixeira, and L. Bosio, J. Chem. Phys. 87, 2231 (1987).

[10] M. A. Floriano, E. Whalley, E. C. Svensson, and V. F. Sears, Phys. Rev. Lett. 57, 3062 (1986).

[11] D. D. Klug, C. A. Tulk, E. C. Svensson, and C.-K. Loong, Phys. Rev. Lett. 83, 2584 (1999).

[12] J. S. Tse, J. Chem. Phys. 96, 5482 (1992).

[13] J. S. Tse, D. D. Klug, C. A. Tulk, I. Swainson, E. C. Svensson, C.-K. Loong, V. Shpakov, V.R. Belosludov, R. V. Belosludov, and Y. Kawazoe, Nature (London) 400, 647 (1999).

[14] C. A. Tulk, D. D. Klug, R. Branderhorst, P. Sharpe, and J. A. Ripmeester, J. Chem. Phys. 149, 8478 (1998).

[15] G. P. Johari, Phys. Chem. Chem. Phys. 2, 1567 (2000).

[16] A. Bizid, L. Bosio, A. Defrain, and M. Oumezzine, J. Chem. Phys. 87, 2225 (1987).

[17] M.-C. Bellisent-Funel, L. Bosio, A. Hallbrucker, E. Mayer, and R. Sridi-Dorbez, J. Chem. Phys. 97, 1282 (1992).

[18] A. K. Soper, W. S. Howells, and A. C. Hannon, Rutherford Appleton Laboratory Report No. RAL-89-046, 1989.

[19] A. K. Soper, Chem. Phys. 202, 295 (1996).

[20] A. K. Soper, Chem. Phys. 258, 121 (2000).

[21] A. K. Soper, Mol. Phys. 99, 1503 (2001).

[22] D. T. Bowron, J. L. Finney, and A. K. Soper, J. Phys. Chem. B 102, 3551 (1998).

[23] D. T. Bowron, A. K. Soper, and J. L. Finney, J. Chem. Phys. 114, 6203 (2001).

[24] W.F. Kuhs, J. L. Finney, C. Vettier, and D. V. Bliss, J. Chem. Phys. 81, 3612 (1984).

[25] C. Lobban, Ph.D. thesis, University of London, 1998.

[26] O. Mishima, L.D. Calvert, and E. Whalley, Nature (London) 314, 393 (1984).

[27] H. F. J. Savage and J. L. Finney, Nature (London) 322, 717 (1986).

[28] A. K. Soper and M. A. Ricci, Phys. Rev. Lett. 84, 2881 (2000). 